\title{
The impact of the major causes of death on life expectancy in China: a 60-year longitudinal study
}

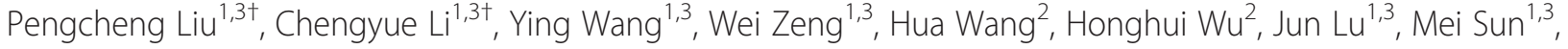
Xiaohong Li $i^{1,3}$, Fengshui Chang ${ }^{1,3}$ and Mo Hao ${ }^{1,3^{*}}$

\begin{abstract}
Background: In the 12th Five-Year Plan, the Chinese government set the goal of increasing life expectancy by one year. The purpose of this study is to examine the impact of major causes of death on the life expectancy of the Chinese people between 1950 and 2010 and predict changing trends to identify major issues requiring future attention.

Methods: A continuous database organised by population and death data on diseases by age group between 1950 and 2010 were created from A Province in Eastern China. The diseases were classified into four categories by the International Classification of Diseases-10 (ICD-10): infectious and parasitic diseases, chronic diseases, accidental injuries, and maternal diseases. Potential gains in life expectancy (PGLEs) were applied to reflect the impact on life expectancy caused by deaths from various diseases, by using the cause-eliminated life table.

Results: The PGLEs of infectious and parasitic diseases decreased from 15.59 years in 1950, to 0.07 year in 2010, and have remained low since 2000. However, the PGLEs of chronic diseases increased from 8.70 years in 1950, to 13.36 years in 2010, and indicated an increasing future trend. The two opposite trends exhibited a 'scissors-like difference'. The proportion of accidental injuries and maternal diseases in the death spectrum was low. The PGLEs of accidental injuries decreased from 2.95 years in 1950, to 0.86 year in 2010, maintaining a low level, while the PGLEs of maternal diseases dropped from 0.56 to 0.002 year during the same period, approaching zero.
\end{abstract}

Conclusions: The findings of this study provide useful information, which could contribute to a more effective allocation of public health programmes. In recent years, chronic diseases and accidental injuries have emerged as major factors influencing life expectancy. Primary and secondary prevention actions, such as public education, modification of behaviours, and introduction of safety measures should be emphasised in efforts to promote life expectancy. The morbidity and mortality rates of infectious, parasitic, and maternal diseases should be maintained at low levels.

Keywords: China, Infectious and parasitic diseases, Chronic diseases, Accidental injuries, Maternal diseases, Impact, Life expectancy, Potential gains in life expectancy (PGLEs), Mortality data

\section{Background}

Life expectancy provides an estimate of the average expected life span under certain conditions, based on current mortality. It is one of three comprehensive indicators reflecting an individual's health, social and economic status, and quality of life [1]. It is also the most

\footnotetext{
* Correspondence: haomo03@fudan.edu.cn

${ }^{\dagger}$ Equal contributors

'Research Institute of Health Development Strategies, Fudan University,

Shanghai 200032, China

${ }^{3}$ The Innovation Center for Social Risk Governance in Health, Shanghai 200032, China

Full list of author information is available at the end of the article
}

representative and comprehensive index to judge the social economy and healthcare development of a country or region [2]. Therefore, it has been incorporated gradually into the evaluation system of governmental performance. Since the founding of the People's Republic of China (PRC) in 1949, China has markedly improved the health of its people. The life expectancy of Chinese residents has more than doubled, from 35 years of age in 1950 , to 74.8 in 2010 . The maternal mortality rate has decreased from 1,500/100,000 to $30 / 100,000$, and the infant mortality rate has dropped from $200 \%$ to $13.1 \%$ [3].

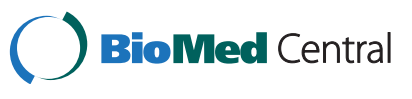

(c) 2014 Liu et al.; licensee BioMed Central Ltd. This is an Open Access article distributed under the terms of the Creative Commons Attribution License (http://creativecommons.org/licenses/by/4.0), which permits unrestricted use, distribution, and reproduction in any medium, provided the original work is properly credited. The Creative Commons Public Domain Dedication waiver (http://creativecommons.org/publicdomain/zero/1.0/) applies to the data made available in this article unless otherwise stated. 
However, changes in living conditions and lifestyles due to the aging of the population and urbanisation and industrialisation have changed China's disease and death spectrum. The United Nations predicted that China's elderly population (over 65 years) would reach 133 million by 2015, 169 million in 2020, and 197 million in 2025 [4]. The Fourth National Health Services Survey of China [5] reported that the morbidity rate of chronic diseases was $19.99 \%$ in 2008, indicating an increase of 10 million new cases on average, every year during the past decade, while the morbidity rate of infectious diseases dropped to $0.27 \%$. According to the Third Death Cause Retrospective Review in 2005, infectious diseases and maternal and infant diseases that accounted for the proportion of total deaths dropped from $27.8 \%$ to $5.2 \%$, while chronic diseases rose from less than $50 \%$ to $82.7 \%$ during 1973-2005. Today, China's disease and death spectrum places chronic diseases as the major cause of death, with some of the major infectious diseases continuing to threaten people's health [6,7].

The Chinese government has attached great importance to the improvement of the population's health. In the 12th Five-Year Plan of China [8], the government set the goal of increasing life expectancy by one year. To achieve this goal, reasonable health policies based on preliminary measures must be developed to understand the major issues.

The purpose of this study is to examine the impact of the major causes of death on the life expectancy of the Chinese people and the changing trends in their health status between 1950 and 2010, which should allow us to provide a clearer perspective of their impacts on life expectancy. We also used historical and current data to predict health trends and changes to identify major issues requiring future attention.

Previous studies [9-13] have used life tables with potential gains in life expectancy (PGLEs) as a research method to determine the impact of fatal diseases that cause death on the life expectancy of a population. However, most of these studies have focused on annual data or data from short time-spans, and do not reflect trends of the impact over longer periods or predict future trends. This study uses the PGLE method that eliminates certain causes of death to determine life expectancies in their absence, and focuses on a 60-year time span. This approach should allow us to reflect clearer trends of the impact of the major causes on life expectancies.

\section{Methods}

\section{Study setting}

We planned to collect population data on deaths caused by various diseases for each year from 1950 to 2010, by age group. However, it was difficult to obtain nationwide data that was continuous and accurate by age group because of the limited information on disease control and prevention, and lags in reporting deaths and disease to the registration system. In addition, it was difficult to coordinate the process of data collection because the management of the population's disease mortality data belongs to different departments of the government.

We selected A Province, which is located in the Eastern Region of China, as the research setting. It is a relatively well-developed area compared to the Middle and Western regions. In 2010, A Province's per capita GDP was 52,664 RMB, and it was ranked fourth among the eight provinces of Eastern China, in per capita GDP [14]. The health developments of A Province exhibited rapid progress in the past 60 years since China was founded [15], and data such as death rates due to diseases have been well preserved. Additionally, our study received support from the Health Department of A Province.

\section{Measures \\ Potential gains in life expectancy (PGLEs)}

Based on the theory of competing risks and the recommendations generated from previous research studies, we selected PGLE, which eliminates certain causes of death as the indicator of their impact on life expectancy. This method permits the analysis of the impacts of several causes of death on the average lifetime of the individuals in a population, taking into account the competing risks acting among them [16]. It has been cited as an ideal method for this type of research [17].

As a quantitative measure, PGLEs can reflect the years of life lost resulting from deaths caused by a certain disease in a specific age group of the population [11]. It is defined as the number of additional years that a person of a certain age would expect to live on average, if the specific cause of death were eliminated [13]. The basic assumption is that if a particular disease that causes deaths is eliminated; those who might have died from the diseases live longer, thereby prolonging the life expectancy of the population. We called the prolonged life expectancy caused by the elimination of deaths from certain diseases the cause-eliminated life expectancy, which can be calculated by building a cause-eliminated life table. The PGLE of a disease in a certain year is calculated as follows: assuming that the cause-eliminated life expectancy is $e_{x}$ and the life expectancy in the presence of all causes of death in the same year is $E_{x}$, then the PGLE in this year, $\Delta E_{x}$ is

$$
\Delta E_{x}=e_{x}-E_{x}
$$

If we use the calculation of the PGLE of heart disease in A Province in 2010 as an example, then the PGLE of heart disease equals the cause-eliminated life expectancy 
of heart disease $e_{2010}$ minus the life expectancy of 2010 $E_{2010}$, yielding a result of 1.68 years.

If we eliminate a larger threat to the population's health, the life expectancy will be extended further and the PGLE will be larger. Therefore, the PGLE is a reasonable way to explain the impact of a certain disease on life expectancy because it can reflect the loss of life expectancy caused by a certain disease and provide a numerical indicator of survival if the disease is eliminated. In addition, this indicator is not affected by the age structure of the population, facilitating comparisons between diseases [18].

\section{Classification of diseases}

Based on the Tenth Revision of the International Classification of Diseases (ICD-10), this study classified the main causes of death into four categories: infectious and parasitic diseases, chronic diseases, accidental injuries (injury and poisoning), and maternal diseases. Although the proportion of deaths due to maternal diseases has been low, the maternal mortality rate was very high worldwide in the early years of the PRC, and declined noticeably between 1950 and 2010. Moreover, the maternal mortality rate is one of three indicators commonly used to evaluate a population's health. Therefore, we assessed the impact of maternal diseases on life expectancy in this study.

Maternal diseases include the complications of pregnancy, childbirth, and the puerperium. Chronic diseases include tumours, haematopoietic organs and immune diseases, mental disorders, nervous system diseases, circulatory-system diseases, respiratory diseases, digestive system diseases, musculoskeletal and connective tissue diseases, and genitourinary system diseases. This study also analysed diseases with high morbidity and mortality rates in China, such as tumours, circulatory-system diseases (including cerebrovascular disease, heart disease, and high blood pressure), and diabetes, accounting for nearly $80 \%$ of all deaths among the entire population in 2010 .

\section{Data sources}

We planned to collect population and death data (1950-2010) on the diseases by age group of A Province, according to the basic requirements of the causeeliminated life table. Age was categorised into the following 19 groups: <1, 1-4, 5-9, 10-14, 15-19, $20-24,25-29,30-34,35-39,40-44,45-49,50-54$, $55-59,60-64,65-69,70-74,75-79,80-84$, and $>85$.

The population data were derived from the 'Statistical Yearbook of A Province' [19], which contains census data. The mortality data on infectious and parasitic diseases before 2004 were taken from the 'Health Statistical Data Collection of A Province' and the 'Acute Infectious Disease Epidemic Information Collection of A Province', and after 2004, were taken from the Direct Network System of Infectious Diseases in China. The mortality data on chronic diseases, accidental injuries, and maternal diseases were obtained from the 'Health Statistical Data Collection of A Province'.

Since several years of data from 1950 to 2010 were missing, these data were simulated through a time series model based on historical data. Finally, we created a continuous database that spanned 60 years.

\section{Data analysis}

We created a database of different disease types in Excel 2010. Data analysis and time series modelling were conducted using Excel 2010 and Eview software. The PGLEs were calculated from the life table and the cause-eliminated life table.

\section{Results}

\section{Trends of life expectancy and disease mortality}

In the past 60 years, the life expectancy and disease mortality of A Province changed substantially. The mortality rates of infectious and parasitic diseases decreased from $848.3 / 100,000$ to $5.2 / 100,000$; accidental injuries from 177.1/100,000 to 39.8/100,000; and maternal diseases from $280.3 / 100,000$ to $6.24 / 100,000$. However, the mortality rates of chronic diseases increased from 391.2/100,000 to $691.3 / 100,000$. The life expectancy of A Province increased markedly from 51.9 to 78.5 . It should be noted that tumours and circulatory-system diseases accounted for most of the deaths due to chronic diseases. For example, in 2010, the mortality rates, respectively, of tumours and circulatory-system diseases were 193.13/ 100,000 and 268.86/100,000 (cerebrovascular disease $151.75 / 100,000$ and heart disease 110.14/100,000).

\section{Historical trends of PGLEs from 1950-2010}

In 1950, the PGLEs of infectious and parasitic diseases, chronic diseases, accidental injuries, and maternal diseases were 15.59, 8.70, 2.95, and 0.56 years, respectively, in A Province (Figure 1). In the years shortly after the founding of the PRC, the PGLEs of infectious and parasitic diseases were the largest, followed by chronic diseases, accidental injuries, and maternal diseases. At the time, the nutrition, living environment, and health conditions of the residents were very poor. Infectious and parasitic diseases were widespread, which led to high death rates in the population. At the same time, the mortality rates of chronic diseases and accidental injuries were very low.

However, with improvements in disease control and prevention, living environment, and nutritional status, the morbidity and mortality rates of infectious and parasitic diseases declined rapidly. The PGLEs also decreased from 15.59 years in 1950, to 0.07 year in 2010, and have 


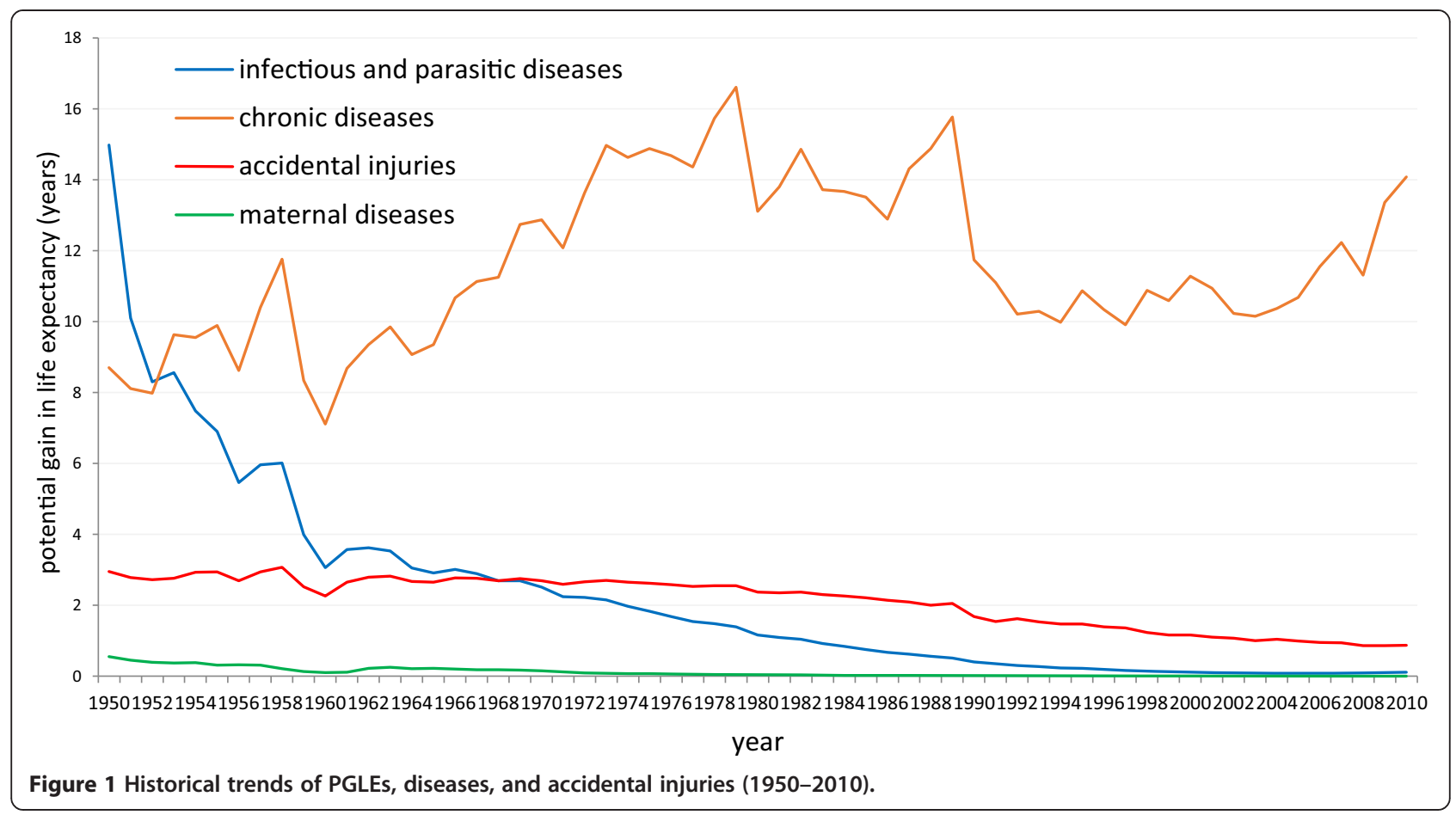

remained low in recent years since 2000. In contrast, the PGLEs of chronic diseases increased from 8.70 years in 1950 , to 14.07 years in 2010, and have exhibited an increasing trend because lifestyle changes have led to rising morbidity and mortality rates of chronic diseases. The two opposite trends in morbidity and mortality are evidenced by the 'scissors-like difference' between the PGLEs.

Compared to the sharp changes in the trends of infectious and parasitic diseases and chronic diseases, the proportion of accidental injuries and maternal diseases in the death spectrum is low. In recent years, mortality rates dropped annually because of positive prevention and control measures. The PGLEs of accidental injuries decreased from 2.95 years in 1950, to 0.89 year in 2010, maintaining a low level, while the PGLEs of maternal diseases dropped from 0.56 to 0.0018 in the same period, approaching zero.

This trend suggests the need to develop measures to prevent and control chronic diseases and accidental injuries as a way to improve people's life expectancy, while maintaining low death rates due to infectious, parasitic, and maternal diseases.

We further examined the PGLEs of several types of chronic diseases in order to understand the impact of the major chronic diseases on life expectancy (Figure 2). The trends in the PGLEs of the major chronic diseases were similar to the entire group of chronic diseases. The diseases with high morbidity rates, such as cerebrovascular disease, heart disease, hypertension, and diabetes, showed an increasing trend year by year. The rising rate of cerebrovascular disease is the largest, from 0.219 to 3.294 during 1950-2010. The PGLEs of hypertension changed the least, from 0.0004 to 0.097 ; heart disease and diabetes increased to a lesser extent.

Unlike the trends of the entire group of chronic diseases, the control of tumours improved in the past 30 years. The PGLEs of the tumours gradually increased at the beginning, but flattened out and even decreased in recent years. This trend also reflects the death trends in the major types of chronic diseases in the population.

\section{Predicting trends of PGLEs for 2011-2020}

Based on the trends in total mortality and the mortality of each age group during 1950-2010, we used time series modelling to fit the trend curve of disease mortality (goodness of fit $>0.8$ ). We predicted the disease mortality trend in each age group during 2011-2020 in accordance with the fitting curve. Then, we calculated the PGLEs of different types of diseases using the cause-eliminated life table from 2011 to 2020, which were regarded as 'the natural trend of PGLEs'. However, if public health efforts changed in controlling and preventing disease, the trends in the PGLEs would also be expected to change. This study predicted future PGLEs when prevention and control efforts changed, using the mortality of different types of diseases as the index of control. We assumed a certain percentage decline in the death rates because of stronger prevention and control measures or a certain percentage rise in the death 


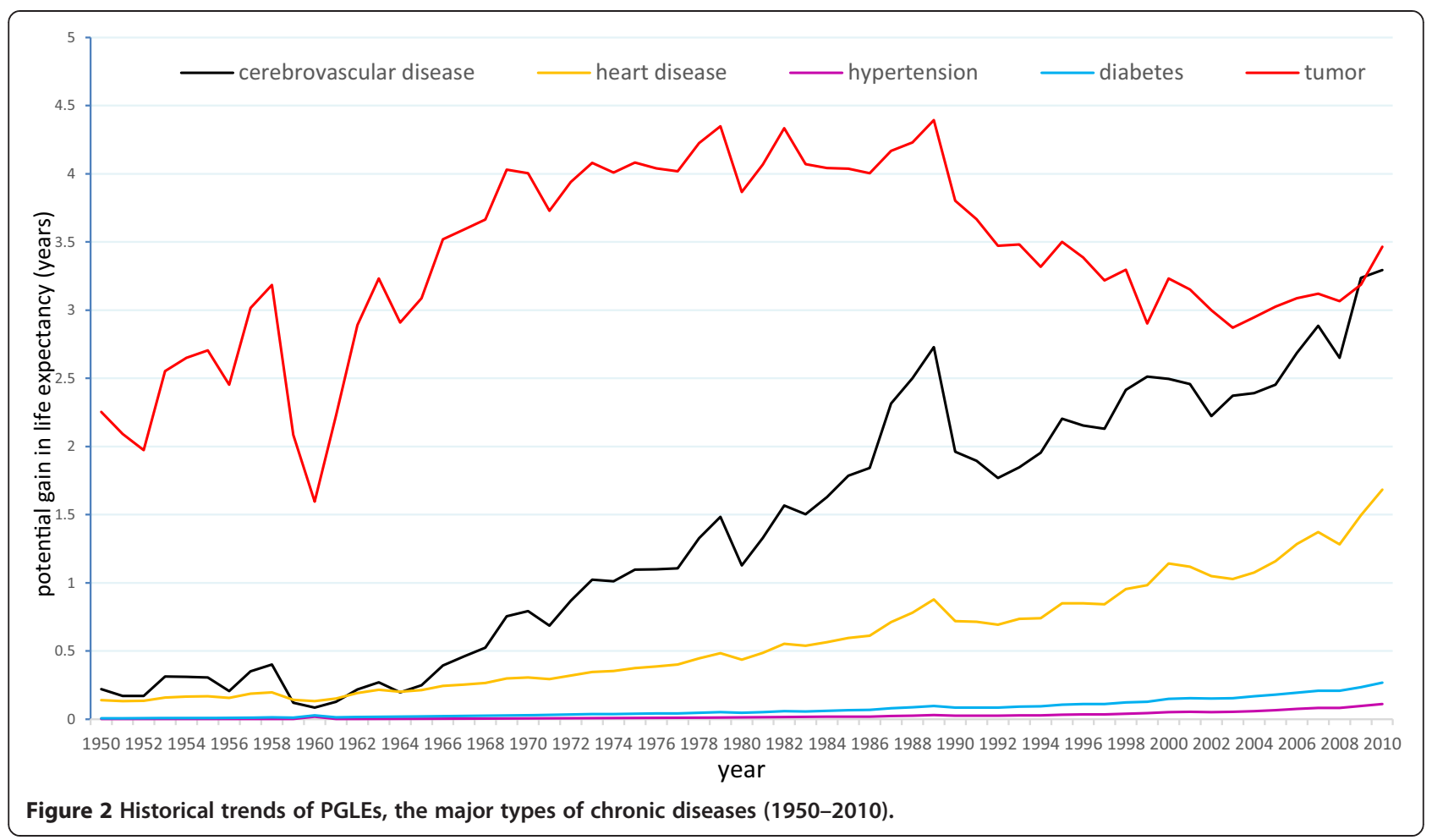

rates resulting from weaker prevention and control efforts. We used stronger prevention and control measures to project future PGLEs in the following example. Based on the fitting curve of the disease mortality trend during 2011-2020, we assumed that the mortality rate of a disease in 2020 was A, under the natural trend, and that stronger prevention and control measures led to a decline in the mortality rate by $\mathrm{X} \%$. Then we calculated PGLEs using the cause-eliminated life table assuming a mortality rate of $A^{*}(1-X \%)$, which would be the result when prevention and control efforts were increased. The decrease and increase percentages in the death rates were determined by consultation with several practice experts from public health centres. Based on their work experience, the consultants suggested an interval for the changes in the percentages, with a $10 \%$ increase as the upper limit and a 10\% decrease as the lower limit. In this study, we used a $10 \%$ change in the death rate as an example to show the trend changes in the PGLEs.

If the intensity of public health efforts to prevent and control the rate of chronic diseases remains unchanged from 2011 to 2020, PGLEs will continue to increase to 18.96 years in 2020 (Table 1). Likewise, if the intensity strengthens or weakens by $10 \%$, PGLEs will be 17.06 or 20.85 in 2020. There will be large differences in PGLEs when natural trends are compared with stronger and weaker efforts. If the current intensity of efforts to prevent and control accidental injuries does not change during 2011-2020, then the PGLEs will continue to decrease to 0.657 year by 2020; and if the efforts increase or decrease by $10 \%$, the PGLEs will be 0.617 or 0.707 year. However, the PGLEs of infectious, parasitic, and maternal diseases will gradually shrink between 2011 and 2020, reaching a very low level. Even if prevention and control measures are increased or decreased by $10 \%$, the PGLEs are expected to show little, if any, change. This forecast suggests that chronic diseases will be the major diseases affecting life expectancy in the future.

The current trends suggest that the PGLEs for cerebrovascular disease will gradually increase during 2011-2020, reaching 7.323 years in 2020 (Table 2). If the prevention and control efforts related to this disease increase or decrease by $10 \%$, the PGLEs will be 6.018 or

Table 1 Predicting outcomes of the PGLEs for accidental injuries and different types of diseases in 2020

\begin{tabular}{cccc}
\hline $\begin{array}{c}\text { Disease } \\
\text { types }\end{array}$ & $\begin{array}{c}\text { Natural } \\
\text { trend }\end{array}$ & $\begin{array}{c}\text { Stronger } \\
\text { prevention } \\
\text { measures (a 10\% } \\
\text { decline in the } \\
\text { mortality) }\end{array}$ & $\begin{array}{c}\text { Weaker } \\
\text { prevention } \\
\text { measures (a 10\% } \\
\text { rise in the } \\
\text { mortality) }\end{array}$ \\
\hline $\begin{array}{c}\text { Infectious and } \\
\text { parasitic diseases }\end{array}$ & 0.0386 & 0.0347 & 0.0424 \\
Chronic diseases & 18.96 & 17.06 & 20.85 \\
Accidental injuries & 0.657 & 0.617 & 0.707 \\
Maternal & 0.000048 & 0.000043 & 0.000053 \\
diseases & & & \\
\hline
\end{tabular}


Table 2 Predicting outcomes of the PGLEs for the major types of chronic diseases in $\mathbf{2 0 2 0}$

\begin{tabular}{cccc}
\hline Disease types & $\begin{array}{c}\text { Natural } \\
\text { trend }\end{array}$ & $\begin{array}{c}\text { Stronger } \\
\text { prevention } \\
\text { measures (a 10\% } \\
\text { decline in the } \\
\text { mortality) }\end{array}$ & $\begin{array}{c}\text { Weaker } \\
\text { prevention } \\
\text { measures (a 10\% } \\
\text { rise in the } \\
\text { mortality) }\end{array}$ \\
\hline $\begin{array}{c}\text { Cerebrovascular } \\
\text { disease }\end{array}$ & 7.323 & 6.018 & 8.929 \\
Heart Disease & 3.150 & 2.739 & 3.597 \\
Hypertension & 0.273 & 0.245 & 0.302 \\
Diabetes & 0.670 & 0.603 & 0.738 \\
Tumours & 4.175 & 3.737 & 4.639 \\
\hline
\end{tabular}

8.929 years. The PGLEs for heart disease show the same trend, reaching 3.150 under the current trend in 2020 and approaching 2.739 or 3.597 when disease prevention and control are increased or decreased. The change is smaller for hypertension with PGLEs expected to reach only $0.273,0.245$, and 0.302 , with current, strengthened, or weakened efforts of the public health system.

The PGLEs for diabetes and tumours will increase to 0.670 and 4.175 , respectively, in 2020. Thus, cerebrovascular diseases and tumours will become the major diseases affecting people's life expectancies in the future.

\section{Discussion}

Between the years 1950 and 2010, the PGLEs of infectious, parasitic, and maternal diseases in China continued to decline while those of chronic diseases showed an upward trend. The PGLEs of accidental injuries remained at a high level while exhibiting a downward trend. It is estimated that the major diseases will continue their previous trends under natural tendencies between 2011 and 2020. If prevention and control efforts directed at different diseases are increased to the same extent, their effects on chronic diseases and accidental injuries should improve while their effects on controlling infectious, parasitic, and maternal diseases should not change significantly.

The PGLEs of infectious and parasitic diseases showed a downward trend because the Chinese government strengthened disease prevention and control measures, such as implementing infectious disease surveillance and planned immunisations. Measures taken to eliminate the development and spread of many infectious diseases gradually resulted in a steady decline in mortality [20,21]. However, some diseases previously under control, such as measles and polio, show signs of returning in recent years. Therefore, strategies to control infectious and parasitic diseases should maintain high standards in programmes unremittingly. Preventing the return of infectious diseases that are under control, and the spread of emerging infectious diseases are also a high priority.
The reasons for the decrease in maternal mortality are similar to the reasons for the decrease in infectious and parasitic diseases. In the early days of the PRC, the maternal mortality rate was high because of lack of medicine and harsh childbirth conditions. As maternal health measures were gradually implemented [22,23], hospital delivery was standardised and popularised, comprehensive prevention and care measures for high-risk pregnant women were drastically improved, and the maternal care of the migrant population was strengthened, with the maternal mortality rate showing a steady downward trend. However, the maternal health of the underdeveloped Western Regions continues to be poor, which also requires more attention in the future [24].

In the early years of the PRC, the mortality rate of chronic diseases was relatively low. However, due to the gradual control of infectious, parasitic, and maternal diseases, acceleration of China's urbanisation and industrialisation, rapid growth of environmental pollution and occupational hazards, and changes in people's lifestyles and working conditions, an increase in the prevalence of chronic diseases occurred, and deaths caused by chronic diseases gradually increased, affecting life expectancy.

In terms of the major types of chronic diseases, the impact of the circulatory-system diseases (including cerebrovascular disease, heart disease, and high blood pressure) and diabetes on life expectancy is increasing year by year. The impact of cancer on life expectancy also shows a rising trend, and this situation will continue for the next few years. Therefore, the prevention of circulatory system diseases and cancer are important considerations in future plans to improve people's health. The modification of behaviours, which have been found to be associated with these specific pathologies, such as dietary habits, physical activity and smoking habits, should be improved.

Although deaths resulting directly from hypertension make a small contribution to the decrease in life expectancy, hypertension is a risk factor for many other diseases. Many patients with hypertension usually suffer from heart disease, cerebrovascular disease, and diabetes. According to one study, the risk attributed to hypertension on mortality resulting from a stroke in the Chinese population is 50\% [25]. Therefore, we must attach great importance to the prevention and control of hypertension.

An analysis of tumours by age group found that the mortality of the younger groups is rising, suggesting that deaths caused by tumours tend to be in the younger population. Hence, the targeted population for cancer prevention and control efforts needs to be expanded. Early diagnosis is important in cancer prevention, and secondary preventive actions, such as screening programs 
can be extended to include younger people in order to achieve a reduction in mortality.

The PGLEs related to accidental injuries is decreasing because the overall injury mortality rate is declining, which has been corroborated by similar studies [26]. Although the overall mortality due to accidental injuries decreased, deaths due to motor vehicle accidents have increased sharply [27]. This finding suggests the need to focus on trends in specific types of injuries, in addition to bringing the overall injury mortality rates under control. Many traffic accidents are preventable by information and education; therefore, taking action to control alcohol abuse and the introduction of safety measures (seat belts, helmets, and child seats) are the most effective solutions [16].

This study had several limitations. First, we were unable to access the nationwide database due to its limited availability, and were only able to examine A Province as a sample. Because of the differences in socioeconomic development among the various provinces of China, the results and conclusions of this study might reflect the trends in the well-developed areas of Eastern China, while extrapolations to the entire country must be made carefully.

When making predictions of PGLEs under different levels of disease control and prevention, we assigned disease mortality as an indicator. Whether there are better indicators to guide the actual work is worth exploring.

\section{Conclusions}

It must be pointed out that the hypothesis of the complete elimination of certain diseases that cause death is not realistic. However, we can evaluate the impacts of the diseases on life expectancies and assign the correct weights to the competing risks among the diseases. In the context of a nation's public health, this approach is helpful for making health polices, developing goals, and allocating resources.

In the early stages of the PRC, infectious and parasitic diseases had a huge impact on life expectancy. With the implementation of health improvements, infectious and parasitic diseases were gradually controlled, and the morbidity and mortality rates of maternal diseases were reduced. In recent years, chronic diseases and accidental injuries have emerged as the major factors influencing life expectancy. With current trends, life expectancy would increase by 0.86 year in the next five years according to our projections, which means the goal of increasing life expectancy by one year could not be reached. Therefore, the prevention and control of chronic diseases and accidental injuries should be emphasised in efforts to promote life expectancy to achieve the goal. We should pay more attention to circulatory-system diseases, tumours, and diabetes. For infectious, parasitic, and maternal diseases with low morbidity and mortality rates, the priority is to maintain these low levels.

\section{Abbreviations}

ICD-10: International Classification of Diseases-10; PRC: the People's Republic of China; PGLE: Potential gain in life expectancy.

\section{Competing interests}

The authors declare that they have no competing interests.

\section{Authors' contributions}

$\mathrm{PL}$ and $\mathrm{CL}$ participated in the design and conceptualisation of the study, acquisition of data, data analysis and interpretation, statistical methods, drafting the manuscript, acquisition of funding, and administrative and material assistance. YW and WZ participated in the data analysis, and drafting the manuscript. HW, HHW, JL, MS, XL, and FC participated in the interpretation and acquisition of data. $\mathrm{MH}$ participated in the design and conceptualisation of study, acquisition of data, drafting the manuscript, acquisition of funding, and supervision. All authors read and approved the final manuscript.

Authors' information

Pengcheng Liu and Chengyue Li are co-first authors.

\section{Acknowledgements}

This study was funded by Innovative Research Team in University from the Ministry of Education of China [IRT_13R11], Program of National Social Science Fund of China [13AZD081], National Natural Science Foundation of China [71373004 and 71303058], the Specialized Research Fund for the Doctoral Program of Higher Education from the Ministry of Education of China [20120071110055], Specialized Fund for The Innovation Centre for Social Risk Governance in Health of Fudan University, Humanities and Social Science Research Project from the Ministry of Education of China [12YJCZH100], and Project of Philosophy and Social Science Development Report from the Ministry of Education of China [11JBGP039].

\section{Author details}

'Research Institute of Health Development Strategies, Fudan University, Shanghai 200032, China. ${ }^{2}$ Health and Family Planning Commission of Jiangsu Province, Nanjing, Jiangsu 210009, China. ${ }^{3}$ The Innovation Center for Social Risk Governance in Health, Shanghai 200032, China.

Received: 26 June 2014 Accepted: 10 November 2014 Published: 20 November 2014

\section{References}

1. Tsai SP, Lee ES, Katz JA: Changes in life expectancy in the United States due to declines in mortality 1968-1975. Am J Epidemiol 1982, 116(2):376-384

2. Tian K: The average life expectancy change of Chinese population and its influence on life insurance. Chinese Insurance 2011, 7:24-27.

3. Li B: Report of the meeting of propaganda and education series on Socialism with Chinese characteristics and the Chinese dream. [http:// www.nhfpc.gov.cn/lbwz/plxfs/201309/54071cc2eb924be684bac31fd96a0e50. shtml]

4. United Nations: World Population Prospects: the 2002 Revision. In [http:// www.un.org/esa/population/publications/wpp2002/WPP2002HIGHLIGHTSrev1.PDF]

5. The ministry of health of China announced the results of the fourth national health services survey. [http://www.moh.gov.cn/mohbgt/s3582/ 200902/39201.shtml]

6. China Internet Network Information Centre: The Ministry of Health released the third death cause retrospective review. [http://www.china. com.cn/zhibo/2008-04/29/content_15028586.htm]

7. Yang GH: Death of Chinese People and Epidemic Levels, Trends, and Distribution of Risk Factors. Beijing: Chinese Peking Union Medical College Press; 2005.

8. The Twelfth Five-year Plan for National Economic and Social Development, the People's Republic of China. [http://www.gov.cn/2011/h/ content_1825838.htm]

9. Sun $H L, L i$ F: Factors influencing the residents' average life expectancy in 2006, Wuhan. Chronic Dis Prev Control China 2008, 16(4):419-420.

10. Hu RY, Gong WW, Pan J: The impact of main cause of death on life expectancy in Zhejiang province, 2010. Dis Surveill 2012, 27(6):485-488. 
11. Wang HG: Study on the Influence of Main Diseases to Life expectancy of Chinese Residents. Peking Union Medical College: Master thesis; 2011.

12. Canudas-Romo V, Liu L, Zimmerman L, Ahmed S, Tsui A: Potential gains in reproductive-aged life expectancy by eliminating maternal mortality: a demographic bonus of achieving MDG 5. PLoS One 2014, 9(2):86-94.

13. Elizabeth $\mathrm{A}$, Melonie $\mathrm{H}$, Betzaida T: United States life tables eliminating certain causes of death, 1999-2001. Natl Vitl Stat Report 2013, 9(61):1-128 [http://www.cdc.gov/nchs/data/nvsr/nvsr61/nvsr61_09.pdf]

14. National Bureau of Statistics of China: China Statistical Yearbook (2011). [http://www.stats.gov.cn/tjsj/ndsj/2011/indexch.htm]

15. Xia ZM, Zhou SG: Background materials of Jiangsu health. Health Econ Res 1997, 2:40-43.

16. Conti S, Farchi G, Masocco M, Toccaceli V, Vichi M: The impact of the major causes of death on life expectancy in Italy. Int J Epidemiol 1999, 28(5):905-910.

17. Ferguson B, Restrepo JA, Villamarín A: Estimating potential gains in life expectancy by reducing violent deaths in selected countries. [https:// www.diw.de/documents/dokumentenarchiv/17/diw_01.c.346948.de/ restrepo_conflict_gecc.pdf]

18. Zhao NQ, Chen F: Health Statistics. Beijing: Higher Education Press; 2008.

19. Statistics Information Network of Jiangsu: Statistical yearbook of Jiangsu Province. [http://www.jssb.gov.cn/jstj/jsnj/2010/tjnj.htm]

20. Wen $L B$, Wang $L Y$ : Death trend analysis of acute infectious diseases in Hongkou, Shanghai, 1952-1999. Dis Control J 2001, 5(2):155-156.

21. Zhang GQ: Epidemic Trend Analysis of Infectious Diseases in Shouguang from 1990-2012. Shandong University: Master thesis; 2013.

22. Xue YX: Maternal mortality trends analysis in Changxing from 1993-2004. China's Matern Child Care 2007, 22:1834-1835.

23. Zhu XY, Liu $L R$, Cui ZL: Maternal mortality rate trends and influencing factors of Henan province in 2000-2010. Chinese J Health Stat 2013, 30(2):279-280

24. Wang B, Liu YH, Guo Y: The inequality status of maternal mortality in China from 1996 to 2006. Matern Child Health Care China 2008, 23(13):1759-1762.

25. Liu LS: Summary report about systolic hypertension clinical trials of Chinese elderly. Cardio-Cerebrovasc Dis Chinese Elder 2000, 2(6):365-367.

26. Diao WL, Feng YP, Zhang SJ, Li N, Mu HJ, Xing LY, Pan GW: Injury mortality change analysis of urban and rural residents in Liaoning province in 1973-1975 and 2004-2005. Chronic Dis Preven Control China 2012, 20(5):539-542

27. Gao YL, Chen XF, Ji K, He J, Yi GH, Zhang NM, Wang Z, Deng Y, Liu LL: The change trend of injury death in cause monitoring points of Sichuan Province. J Prev Med Inform 2009, 25(8):633-637.

doi:10.1186/1471-2458-14-1193

Cite this article as: Liu et al:: The impact of the major causes of death on life expectancy in China: a 60-year longitudinal study. BMC Public Health 2014 14:1193.

\section{Submit your next manuscript to BioMed Central and take full advantage of:}

- Convenient online submission

- Thorough peer review

- No space constraints or color figure charges

- Immediate publication on acceptance

- Inclusion in PubMed, CAS, Scopus and Google Scholar

- Research which is freely available for redistribution 